\title{
A study of maternal serum lipids in pregnancies complicated by pre-eclampsia in a cohort of Nigerian women
}

\author{
Yakubu Emmanuel Nyam¹, Ajen Stephen Anzaku², Madziga Isa Gamece ${ }^{3}$, Daru Patrick Haruna ${ }^{1}$ \\ ${ }^{1}$ Department of Obstetrics and Gynaecology, Jos University Teaching Hospital, Jos, Nigeria \\ ${ }^{2}$ Department of Obstetrics and Gynaecology, College of Health Sciences, Bingham University, Karu, Jos Campus, Jos, Nigeria \\ ${ }^{3}$ Nigeria National Petroleum Corporation, Kaduna, Nigeria
}

\section{Email address:}

emayaks75@yahoo.com (Y. E. Nyam)

\section{To cite this article:}

Yakubu Emmanuel Nyam, Ajen Stephen Anzaku, Madziga Isa Gamece, Daru Patrick Haruna. A Study of Maternal Serum Lipids in Pregnancies Complicated by Pre-Eclampsia in a Cohort of Nigerian Women. Science Research. Vol. 2, No. 6, 2014, pp. 150-154. doi: 10.11648/j.sr.20140206.11

\begin{abstract}
Background: Pre-eclampsia is a hypertensive disorder in pregnancy, that has adverse maternal and perinatal outcomes. Abnormal serum lipid levels have been implicated in the pathogenesis of pre-eclampsia, this can give rise to increased oxidative stress and then subsequently, endothelial dysfunction. Methods: This study was a comparative crosssectional study that involved fifty pregnant women that had pre-eclampsia and another fifty healthy pregnant women. Cases and controls were matched for Age in years, Parity, Gestational age in weeks and Body mass index in $\mathrm{Kg} / \mathrm{M}^{2}$. Fasting venous blood samples were obtained and analyzed for serum levels of total cholesterol, triglycerides, low-density lipoproteins (LDL), and high-density lipoproteins (HDL) using enzymatic colorimetric method aided by Cobass C111 Autoanalyser machine. The Data was analyzed using Epi-info version 3.5.4, CDC, Atlanta, USA and $\mathrm{P}<0.05$ was considered significant. Results: Mean age of the case and control groups were $28.86 \pm 4.50$ Years and $29.9 \pm 4.95$ Years respectively $(\mathrm{P}=0.59)$. Statistical analysis revealed no significant difference in terms of total Cholesterol $(\mathrm{P}=0.98)$ and low density lipoprotein $(\mathrm{P}=0.92)$. However, mean triglycerides $(1.95 \pm 0.89 \mathrm{mmol} / \mathrm{L}$ versus $1.02 \pm 0.51 \mathrm{mmol} / \mathrm{L})$ was significantly higher in women with pre-eclampsia compared to the control $(\mathrm{P}=0.0004)$ and a significant reduction in high density lipoprotein $(0.79 \pm 0.30 \mathrm{mmol} / \mathrm{L}$ versus $1.74 \pm$ $0.45 \mathrm{mmol} / \mathrm{L})$ in the case group $(\mathrm{P}=0.0005)$. Conclusion: In this study, Pre-eclampsia is associated with elevated serum triglycerides and reduced high density lipoprotein. These findings may serve as screening markers for preeclampsia.
\end{abstract}

Keywords: Pre-Eclampsia, Serum Lipids, Total Cholesterol, Triglycerides, High Density Lipoprotein, Low Density Lipoprotein

\section{Introduction}

Pre-eclampsia is a multi-systemic pregnancy related medical disorder associated with maternal and fetal morbidity and mortality ${ }^{1}$. Pre-eclampsia is characterized by onset of hypertension $(\geq 140 / 90 \mathrm{mmHg})$ and proteinuria ++ or more in a strip dip test or total protein excretion of 300 $\mathrm{mg} / \mathrm{dL}$ in a 24-hour urine collection, occurring after 20 weeks of gestation and beyond ${ }^{2}$. In developing countries where access to health care is limited, pre-eclampsia is a major cause of maternal morbidity and subsequent mortality and it complicates approximately $5-7 \%$ of pregnancies ${ }^{3}$. In Nigeria, hypertensive disorders in pregnancy and it's complications accounts for $17 \%$ of maternal deaths, it was found to be the leading cause of maternal death in Gombe, North-eastern Nigeria, accounting for $24.2 \%^{3,4}$. Elsewhere, about $11 \%$ of maternal deaths were attributed to pre-eclampsia and eclampsia in Bangladesh ${ }^{5}$. It is also associated with poor fetal outcomes including premature delivery, intrauterine growth restriction and intrauterine foetal death ${ }^{1,2,3}$.

Despite considerable research, the exact aetiology and pathogenesis of this age-old and life threatening medical condition remains unclear, ${ }^{2}$. Until date, endothelial dysfunction in the placental vascular network is considered as the most widely accepted theory for the aetiology and pathogenesis of Pre-eclampsia ${ }^{6,7}$. Other theories include the genetic theory, immune response theory, and oxidative stress 
theory ${ }^{6},{ }^{7}$. Previous researches suggested that maternal predisposition to preeclampsia may be explained by altered maternal serum lipid profile but the findings were inconsistent ${ }^{8-10}$.

The association between alterations in serum lipid profile and pre-eclampsia is a relatively new concept, although there is a pronounced physiological hyperlipidaemia, involving serum levels of triglycerides and cholesterol in normal pregnancy ${ }^{11}$. However, women with pre-eclampsia tend to display additional alterations in serum lipids, and this may be reflection of disordered lipid and lipoprotein metabolism ${ }^{11,12}$. Studies in the field of cardiovascular research found serum lipids to have direct effects on the endothelial function, suggesting that abnormal serum lipid profile are associated with endothelial dysfunction ${ }^{13}$. The rise in serum lipid parameters is reported to be susceptible to oxidation which may cause increased oxidative stress, generate free radicals and contribute to endothelial damage and this cascade of events is associated with an increased risk of occurrence of pre-eclampsia ${ }^{14}$. There is a growing evidence that preeclampsia is increased in women with elevated level of oxidized low-density lipoproteins and triglycerides in preeclamptic women ${ }^{15}$.

In view of above information, this study was designed to study the maternal serum levels of high density lipoprotein (HDL)-cholesterol, Low density lipoprotein (LDL)cholesterol, triglycerides and total cholesterol in Preeclamptic women and was compared with that of normal pregnant women in a Nigerian obstetric population.

\section{Materials and Methods}

This study was conducted at the Jos University Teaching Hospital, Plateau state, Nigeria. The sampled populations were recruited from the antenatal clinic, antenatal ward and labour ward of the hospital. A total of one hundred pregnant women with singleton pregnancies were placed into two groups (case and control groups). Each of the groups consisted of fifty subjects, who were recruited consecutively by a non-probability convenient sampling method. The two groups were matched for age in years, parity, gestational age in weeks and body mass index in $\mathrm{Kg} / \mathrm{M}^{2}$. The criteria for the diagnosis of pre-eclampsia was based on blood pressure measurement, using a mercury sphygmomanometer, of at least $140 \mathrm{mmHg}$ and $90 \mathrm{mmHg}$ systolic and diastolic blood pressure respectively, detected after $20^{\text {th }}$ weeks of gestation, taken in sitting position, done twice, at least 6 hours apart. The diagnosis of proteinuria was based on urine dipstick of at least $2+$ after the $20^{\text {th }}$ week of gestation. A blood pressure measurement of at least $160 \mathrm{mmHg}$ systolic and $110 \mathrm{mmHg}$ diastolic pressures, alongside proteinuria of at least $3+$, after the $20^{\text {th }}$ week of gestation, was considered severe Preeclampsia.

Five milliliter $(5 \mathrm{~mL})$ of fasting blood sample was collected via the ante-cubital vein from the sampled populations, by the use of sterile disposable $5 \mathrm{~mL}$ syringe and each of the samples was transferred immediately into an appropriately labeled plain bottle container and was allowed to clot at room temperature. The samples were thereafter centrifuged at 4000 revolutions per minute (rpm) for 10 minutes and each serum sample separated was transferred into a new appropriately labeled sample container (cryovials) and stored at $-70^{\circ} \mathrm{C}$ until the time of analysis for serum lipids. The biochemical analysis was done for total cholesterol (TC), triglycerides (TG), high density lipoprotein (HDL) and low density lipoprotein (LDL) using the appropriate reagent kits for Randox and Cobass $\mathrm{C}-111^{\circledR}$ Autoanalyser machine was used to run the blood samples. The Low density lipoprotein was calculated using the Friedewald's formula ${ }^{16}$.

The data obtained was analyzed using Epi-info version 3.5.4 from CDC Atlanta, Georgia. The means, Standard deviation and their variations were determined and the student- $t$ test was used to test for the mean difference. The level of significance was set at $\mathrm{P}<0.05$. In Jos, University Teaching Hospital(JUTH) the following reference ranges for lipid profile were adopted; Total Cholesterol 2.5-6.5 mmol/L, Triglycerides $0.5 \quad-1.7 \quad \mathrm{mmol} / \mathrm{l}$, HDL-cholesterol $1.1-1.7$ $\mathrm{mmol} / 1$, and LDL cholesterol $<2.33 \mathrm{mmol} / \mathrm{L}$.

Ethical Consideration: This study was undertaken after due approval from the ethical committee of the Jos University Teaching Hospital (JUTH). Informed consent was duly obtained from all subjects who participated in the study.

\section{Results}

Table 1. Socio-demographic Characteristics of the Subjects studied

\begin{tabular}{|c|c|c|}
\hline Characteristics & Frequency & Percentage \\
\hline \multicolumn{3}{|l|}{ Age Group in Years } \\
\hline$\leq 18$ & 2 & 2.0 \\
\hline $19-23$ & 11 & 11.0 \\
\hline $24-28$ & 37 & 37.0 \\
\hline $29-33$ & 31 & 31.0 \\
\hline $34-38$ & 15 & 15.0 \\
\hline$\geq 39$ & 4 & 4.0 \\
\hline Total & 100 & 100 \\
\hline \multicolumn{3}{|l|}{ Educational Level } \\
\hline None & 3 & 3.0 \\
\hline Primary & 13 & 13.0 \\
\hline Secondary & 37 & 37.0 \\
\hline Tertiary & 47 & 47.0 \\
\hline Total & 100 & 100 \\
\hline \multicolumn{3}{|l|}{ Ethnicity } \\
\hline Hausa/Fulani & 17 & 17.0 \\
\hline Igbo & 5 & 5.0 \\
\hline Yoruba & 8 & 8.0 \\
\hline Ethnics from Plateau & 50 & 50.0 \\
\hline Others * & 20 & 20.0 \\
\hline Total & 100 & 100 \\
\hline \multicolumn{3}{|l|}{ Occupation } \\
\hline Housewife and Unemployed & 27 & 27.0 \\
\hline Student & 8 & 8.0 \\
\hline Trader & 24 & 24.0 \\
\hline Artisan & 4 & 2.0 \\
\hline Civil Servant & 36 & 36.0 \\
\hline Others $* *$ & 1 & 3.0 \\
\hline Total & 100 & 100 \\
\hline
\end{tabular}

Other Ethnicity*: Idoma, Tiv, Bassange, Igala, Eggon, Ribina Other Occupations**: Bankers, Caterer, Lawyers, and Nurses 
The age of the subjects in this study ranged from 18 to 39 years and the age group with the highest frequency was 2428 years which constituted $37 \%$ of entire studied subject. The mean age among the pre-eclamptic subjects was $28.86 \pm 4.50$ years, while the mean age for the non-preeclamptics group was $29.90 \pm 4.5$ years $(\mathrm{P}=0.586)$. Most of the pre-eclamptic women were primigravidae [31(62.0\%)] while $29(38.0 \%)$ were Multigravidae. The mean BMI for the case and control group was $24.36 \pm 3.96 \mathrm{Kg} / \mathrm{m}^{2}$ and $25.24 \pm 3.44 \mathrm{Kg} / \mathrm{m}^{2}$ respectively $(\mathrm{P}=0.933)$. The mean gestational age for the case and control group was $33.20 \pm 0.48$ weeks and $34.20 \pm$ 0.50 weeks respectively $(\mathrm{P}=0.796)$. Table 1 shows the socio- demographic characteristics of the study population.

Table 2 shows the mean serum lipids among the case and control groups. The mean serum level of triglyceride $(1.95 \pm$ 0.89 versus $1.02 \pm 0.51$ ) was significantly higher in the preeclamptic group compared to the controls $(\mathrm{P}=0.0004)$. Also the mean serum level of high density lipoprotein $(0.79 \pm 0.30$ versus $1.74 \pm 0.45)$ was significantly lower among the preeclamptics compared with the controls $(\mathrm{P}=0.0005)$. Other lipid parameters consisted of total cholesterol and lowdensity lipoprotein appeared not to be different among the two groups with P-values of 0.976 and 0.918 respectively.

Table 2. Bivariate Analysis of Serum Lipids by Groups (Case and Controls)

\begin{tabular}{llll}
\hline \multirow{2}{*}{ Factors Mean } & N $(\%) / M e a n \pm$ SD & & \multirow{2}{*}{ Student t- test } \\
\cline { 2 - 4 } & Pre-Eclamptic & Non Pre-Eclamptic & 0.30 \\
\hline Level of total Cholesterol & $4.54 \pm 0.98$ & $4.60 \pm 1.04$ & 3.94 \\
Serum Level of Triglycerides & $1.95 \pm 0.89$ & $1.02 \pm 0.51$ & 12.48 \\
Serum Level of HDLc & $0.79 \pm 0.30$ & $1.74 \pm 0.45$ & 0.976 \\
Mean calculated Levels of LDLc & $1.85 \pm 0.38$ & $1.90 \pm 0.39$ & 0.0004 \\
\hline
\end{tabular}

\section{Discussion}

The results of this study showed that the age group of the participants fell between 18-39 years. This therefore represents a population of young and middle age women who may not have confounding age-related altered serum lipids usually seen in the elderly. Also most women who had preeclampsia in this study, were primigravidae (i.e. $62 \%$ ), this is not unusual as this type of hypertensive disorder is commoner in women pregnant for the first time. ${ }^{2}$

We also observed hypertriglyceridemia in women with pre-eclampsia $(1.95 \pm 0.89 \mathrm{mmoI} / \mathrm{L})$ compared with the normal healthy pregnant women. This is in keeping with findings from a study that was done in a rural setting of Nigera $^{17}$. It is worthy of note that relative to normal pregnancies, women with pre-eclampsia have marked increases in serum triglycerides ${ }^{18,19}$. It has been suggested that hypertriglyceridaemia in pregnant women between 28 and 32 weeks could be predictive of preeclampsia ${ }^{20}$. The rise in this serum lipid parameter is susceptible to bio-oxidation which may cause increase oxidative stress, generate free radicals and contribute to endothelial damage and propagate the pathogenesis of preeclampsia. This damage usually begins as placental vascular disorder involving the placental spiral arteries and subsequent thrombosis which triggers endothelial dysfunction.

A high level of triglycerides among women with preeclampsia was also reported in studies by other researchers in other countries $5,21,22$. However, studies by Zinat $\mathrm{B}$ et al and Chanvitya $\mathrm{P}$ et al found no significant difference in the level of triglycerides between preeclamptics and non-pre-eclamptics ${ }^{11,23}$. This may be due to differences between their study designs with this study. Also, Ray et.al reported that pregnant women who had elevated serum triglycerides had twice the risk of pre-eclampsia, studies that adjusted for confounders (Age, BMI and Parity) indicated that the risk was four times higher, compared with women with normal triglycerides ${ }^{18}$.

Conversely, this study also found that high density lipoprotein (HDLC) was significantly lower among the preeclamptic group $(0.79 \pm 0.30 \mathrm{mmoI} / \mathrm{L})$ compared with the control group $(1.74 \pm 0.45)$. This finding is similar to work done by Vanderjagt et.al ${ }^{4}$, Abubakar et.al ${ }^{17}$, Islam NAF et.al ${ }^{4}$ and Musleh UK et.al ${ }^{22}$. However, Chanvitya $\mathrm{P}$ et.al ${ }^{23}$ conducted a cross - sectional study but did not find significant difference in serum lipid components among preeclampsia and non-pre-eclampsia studied groups. Also Zinat B. et.al ${ }^{11}$ in a cross-sectional study reported significant difference in the HDL alone among women with preeclampsia. These variant findings may be due to differences in races and nutrition. HDL is an antiatherogenic lipoprotein while atherogenicity is mainly caused by LDL lipid component.

The reduced HDL observed may be useful in understanding the pathogenesis of pre-eclampsia in this regard and its implication for the well-being of the patient with regard to possible risk of cardiovascular complications which could occur considering the cardio-protective function of HDL-c. In this regard, these findings further suggest the need to follow-up women with pre-eclampsia up to the end of the puerperal period. This is to ascertain whether the observed reduction in the serum HDL and a rise in serum triglyceride in this study have a persistent or temporal relationship.

During normal pregnancy HDL fraction have been reported to be elevated or within normal limits ${ }^{11,24}$. However, in this study, a decrease in HDL was observed among the group of pregnant women with pre-eclampsia. This finding may be useful in tracking a group of women who may develop cardiovascular complications such as myocardial infarction, atherosclerosis and coronary heart disease. A relationship between this reduction in HDL, a rise in serum triglycerides and development of this cardiovascular complications may in addition be established, when further 
studies, follow-up of this women has found a persistent reduction in HDL-c and rise in serum triglycerides. Other lipid components such as LDL-c and total cholesterol were within normal limits and no significant difference was found between the two studied groups and this was corroborated by other studies done elsewhere ${ }^{23,24}$.

\section{Study Limitations}

There is a potential for selection bias in an attempt to match for the covariates that may affect serum lipids. Furthermore, not all the covariates would have been taken into account. It is difficult to establish a causal, casual or effect of the disease on the serum lipids analysed. The mechanism of selecting the subjects did not take into account the type of food and a cytogenetic effect is considered weakness of this research.

\section{Strengths of the Study}

The ability to control for confounders has made the studied population homogeneous. The blood samples for the serum lipids were obtained when the women were in a fasting state. Also women with diabetes mellitus, chronic hypertension and women on medications that may influence lipid profile were excluded from the study, as this may have impact on lipid levels and give false impression of the overall assessment.

\section{Challenges}

Some of the participants with mild pre-eclampsia were lost out as they did not keep to appointment for a fasting blood sample collection. This was not so for the consenting women with severe Pre-eclampsia because they were all place on hospital admission for stabilization and possible delivery. It was challenging to have the pregnant women stay in a fasting state before collection of blood sample.

\section{Conclusion}

This study showed a significant high level of triglycerides and a lower serum level of high density lipoprotein among pre-eclamptic women compared with those without preeclampsia. It is difficult to infer from this study, if this finding was due to a cause of preeclampsia or as result of effect of pre-eclampsia. Therefore to make either conclusion, at this point remain a subject of further research. A follow-up plan in the course of pregnancy and thereafter is necessary to establish a possible causal relationship in the pathogenesis of preeclampsia. This finding may help in the understanding of pathogenesis as well as developing strategies for the prevention and early diagnosis of pre-eclampsia.

\section{Acknowledgement}

We wish to acknowledge the immense contribution of Dr. Lucius Imoh, a Consultant chemical pathologist in JUTH, for his contribution and support during the laboratory analysis. We also appreciate Mr. Rotimi, Mr. Mangai and Mr. Bitrus (all laboratory scientists), for also giving us the necessary laboratory supports to undertake the biochemical analysis.

\section{Future Research Direction}

Subsequent studies in this area, could further follow-up this cohort of women in the puerperal and post-puerperal period, to find out if the derangement in the serum lipids is temporal or persistent. This will also provide valuable information on those likely to develop cardiovascular complications in view of the increase in the serum triglycerides and reduction in the high density lipoprotein that was seen from this study.

\section{References}

[1] Dutta DC. Hypertensive disorders in Pregnancy. In: Hiralal K(ed) Textbook of Obstetrics. $7^{\text {th }}$ edition new central book agency Kolkata, India. 2011; 17:219-20.

[2] Jason JS, Waugh, Maria CS. Hypertensive disorder. In: Edmonds DK(ed) Dewhurst's Textbook of Obstetrics and gynaecology, $8^{\text {th }}$ edition. Wiley- Blackwell Publishing 2007; $101-2$.

[3] Abudu O.O, Afolabi B.B. Pregnancy induced hypertension, Pre-eclampsia and Chronic hypertension. In: Akin Agboola (Ed), Textbook of Obstetrics and Gynaecology for Medical Students. $2^{\text {nd }}$ edition; Heinemann Educational Books. 2006:350-51.

[4] Vanderjagt DJ, El-Nafaty AU, Melah GS et.al High density lipoprotein and homocysteine levels correlate inversely in preeclamptics women in Northern Nigeria. Acta Obstet Gynaecol Scand J 2004; 83: 536-42.

[5] NAF Islam, MAR Chodwury, GM Kibria et.al Study of serum lipid profile in pre-eclampsia and Eclampsia. Faridpur Med coll J 2010;5:56-9.

[6] Siddiqui IA. Maternal seum lipids in women with Preeclampsia. Ann Med Health Sci Res 2014;4:638-41.

[7] Young BC, Levine RJ, Karumanchi SA. Pathogenesis of Preeclampsia. Annu Rev Pathol 2010,5:173-92.

[8] Enquobahrie DA, Williams MA, Buttler CL, et.al Maternal plasma lipid concentration in early pregnancy and risk of preeclampsia. Am J Hypertens 2008; 17: 74-81.

[9] Belo L, Caslake M, Gaffney D et.al Changes in LDL size and HDL concentration in normal and preeclamptics pregnancies. Atherosclerosis 2002; 162:425-32.

[10] Brown MA, Lindheimer MD, de Sweit M. The classification and diagnosis of the hypertensive disorder of pregnancy. Statement from the international society for the study of hypertension in pregnancy (ISSHP) Hypertens Pregnancy 2001; 20:9-14.

[11] Zinat B, Iffat A, Shah ABS. Association between lipid profile and Preeclampsia. Ibrahim card Med J 2011; 1:4144. 
[12] Hubel CA, Roberts JM. Hyperuricaemia and xanthine oxidase in preeclampsia revisited Am J Obstet Gynaecol 1996; 174:288-91.

[13] Sima AV, Stancu CS, Simionescu M, Vascular endothelium in atherosclerosis. Cell Tissue Res 2009; 335:191-203.

[14] Lima VJ, Andrade CR et.al Serum Lipid levels in Pregnancies complicated by Preeclampsia. Sao Paulo Med J 2011; 129:7376.

[15] Hiralal K. Aetiopathogenesis of Pre-eclampsia In: Milind RS (Ed), Hypertensive disorders in Pregnancy. $1^{\text {st }}$ Edition. Jaypee Brothers Medical Publishers. 2007; 5:39-46.

[16] Friedwald WT, Levy RI, Fredrickson DS. Estimation of the concentration of Low-density lipoprotein cholesterol in plasma, without use of the ultracentrifuge. Clin Chem 1972; $18: 499-502$

[17] Abubakar A. Mabrouk M. Girei AB et.al Lipid profiles and Platelet counts of pre-eclamptic women in selected rural areas of Nigeria. Web Med Central Physiology.2011:2(8) WMCOO2121.

[18] Ray JG, Diamond P, Singh G, Bell CM. Brief overview of maternal triglycerides as a risk factor for pre-eclampsia. BJOG 2006; 113:379-86.
[19] Kalar MU, Kalar N, Mansoor F, Malik AR, Lessley T, Kreimer $\mathrm{S}$ et al. Preeclampsia and lipid levels - a case control study. International Journal of Collaborative research in internal Medicine and Public Health 2012; 4: 1738 - 45.

[20] Ziaei S, Bonab KM, Kazemnejad A. Serum lipid levels at 2832 weeks gestation and hypertensive disorders. Hypertens Pregnancy 2006; 25(1):3-10.

[21] Musa AH, Mairiga AG, Jimeta AA, Daja A. Lipid profile pattern of Preeclamptic and Eclamptic patients attending University of Maiduguri Teaching Hospital. Gynaecol Obstet (Sunnyvale) 2014; 4:217 - 201

[22] Musleh U.K, Nabila K, Farah M. Preeclampsia and lipid levels- a case control study. International Journal of Collaborative research on internal medicine and public health. 2012; 4:1738-44.

[23] Chanvitya P, Boonsri K. Comparative study of serum lipid concentrations in preeclampsia and Normal Pregnancy. J Med Assoc Thai 2008; 91:957-61.

[24] Baksu B, Baksu A, Davas I. Lipoprotein levels in women with preeclampsia and in normotensive pregnant women. J obstet Gynaecol Res 2005; 31:277-82. 